\section{COVID-19 and panic disorder: clinical considerations for the most physical of mental disorders}

Braz J Psychiatry. 2021 Jan-Feb;43(1):110-111

doi:10.1590/1516-4446-2020-1235

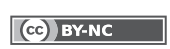

Shortness of breath, choking or smothering sensations, chest pain or discomfort, and fear of dying. Due to the coronavirus disease 2019 (COVID-19) pandemic, our minds have been conditioned to associate these symptoms with a rather severe form of the disease. Although such symptoms could indicate a case of COVID-19, they are also common in panic attacks (PAs) (i.e., the hallmark of panic disorder [PD]), in which respiratory symptoms occur along with other panic symptoms that are mainly physical in nature. ${ }^{1,2}$

Experimental evidence has supported the hypothesis, unique in the realm of mental disorders, that subclinical alterations of basic physical functioning, mainly the respiratory system, may be involved in the pathogenesis of PAs. Patients with PD were thought to have a hyperactive suffocation alarm, which results in a specific behavioral and respiratory hypersensitivity to hypercapnia. Different laboratory challenges that induce hypercapnia (e.g., the Read's rebreathing technique, the prolonged inhalation of $5 \%$ or $7 \%$ of $\mathrm{CO}_{2}$-enriched air, and the double inhalation of a $35 \% \mathrm{CO}_{2}$ and $65 \% \mathrm{O}_{2}$ gas mixture) resulted in higher rates of PAs and respiratory-response abnormalities in PD patients than controls. Moreover, these patients are hypersensitive to various other laboratory respiratory challenges, such as breath-holding, hyperventilation, and a hypoxic challenge test. ${ }^{1,2}$ They suffer from irregular breathing patterns, impaired diaphragmatic breathing with reduced vital capacity, chronic hyperventilation, and a common sensation of difficulty in breathing during activities of daily living. ${ }^{3}$ Finally, PD has remarkable associations with asthma or chronic obstructive pulmonary disease. PD patients also have imbalanced autonomic regulation, reduced heart rate variability, poorer cardiovascular fitness, and higher variability of electrocardiographic QT interval and cardiac repolarization. ${ }^{2}$

Although the source of these features is still unclear, patients with PD experience, from a clinical point of view, a significant burden of respiratory and physical symptoms or discomfort during PAs, as well as in certain environmental situations. Furthermore, the fear of suffocation is one of their primary troubles. Overall, they seem to exhibit greater physical and emotional difficulties in coping with somatic sensations and internal bodily changes than individuals suffering from other anxiety disorders. ${ }^{4}$

Given these peculiar features, PD patients are likely to have distinct difficulty in dealing with a disease like COVID-19, since it may be severe and life-threatening and, most importantly, it may cause various respiratory symptoms, which could lead to respiratory failure. Hence, when PD patients develop COVID-19, a complex clinical picture might emerge that deserves consideration from clinicians involved in managing COVID-19 patients.

First, clinicians should bear in mind that PD patients are particularly hypersensitive, from a biological and psychological point of view, to respiratory stimuli and physical symptoms or sensations. Thus, PD patients with COVID19 may actually perceive more intense respiratory and physical discomfort than other patients, even with mild infection. In more severe cases of COVID-19, it may be especially difficult for PD patients to contend with hypoxia or tolerate various methods of ventilation. Hence, great attention and support should be paid to the respiratory and physical complaints of COVID-19 patients with personal histories of PAs or PD. Furthermore, in COVID-19 patients whose respiratory symptoms are more intense than their physical condition would seem to warrant, clinicians should check for a history of PAs/PD.

Second, some of the most commonly used antipanic medications, particularly paroxetine and clomipramine, have positive effects on respiration and dyspnea. Their abrupt interruption may increase respiratory discomfort and panic symptoms, ${ }^{2}$ which could worsen the condition of patients with respiratory diseases. ${ }^{5}$ Thus, they should not be discontinued in PD patients with COVID-19. Moreover, if a patient's antipanic medication is incompatible with his or her general medical condition, alternative antipanic medications might be considered.

Finally, since PD patients are more likely to experience greater anxiety and fear in response to COVID-19-related respiratory or physical symptoms than other patients, the clinical support of psychiatrists or cognitive behavior therapists might be particularly helpful during this stressful experience.

Of course, patients with other psychiatric disorders may also be at high risk of COVID-19-related distress, and our considerations were not meant to underestimate the expertise of clinicians involved in the COVID-19 pandemic. Our aim was to highlight that the fact that psychiatric aspects deserve attention even during a challenging medical crisis, such as the COVID-19 pandemic, and that PD patients may be particularly challenged by this infection due to the peculiarity of panic pathophysiology.

Giampaolo Perna, ${ }^{1,2}$ iD Daniela Caldirola ${ }^{1,2}$ iD ${ }^{1}$ Dipartimento di Scienze Biomediche, Humanitas University, Milan, Italy. ${ }^{2}$ Dipartimento di Neuroscienze Cliniche, Villa San Benedetto Menni Hospital, Como, Italy.

Submitted Jun 15 2020, accepted Jun 21 2020, Epub Aug 032020. 


\section{Acknowledgements}

The authors would like to thank Enago for the English language review.

\section{Disclosure}

Both authors are scientific consultants for Medibio LTD. GP has served as consultant for Lundbeck and Pfizer.

How to cite this article: Perna G, Caldirola D. COVID-19 and panic disorder: clinical considerations for the most physical of mental disorders. Braz J Psychiatry. 2021;43:110-111. http://dx.doi.org/10.1590/1516-44462020-1235

\section{References}

1 Okuro RT, Freire RC, Zin WA, Quagliato LA, Nardi AE. Panic disorder respiratory subtype: psychopathology and challenge tests - an update. Braz J Psychiatry. 2020;42:420-30.

2 Caldirola D, Perna G. Toward a personalized therapy for panic disorder: preliminary considerations from a work in progress. Neuropsychiatr Dis Treat. 2019;15:1957-70.

3 Grassi M, Caldirola D, Vanni G, Guerriero G, Piccinni M, Valchera A, et al. Baseline respiratory parameters in panic disorder: a metaanalysis. J Affect Disord. 2013;146:158-73.

4 Hoehn-Saric R, McLeod DR, Funderburk F, Kowalski P. Somatic symptoms and physiologic responses in generalized anxiety disorder and panic disorder: an ambulatory monitor study. Arch Gen Psychiatry. 2004;61:913-21.

5 Oleynick C. Recurrent episodes of hypercapnic respiratory failure triggered by panic attacks in a patient with chronic obstructive pulmonary disease. Respir Med Case Reports. 2020;30:101044.

\section{FK506 binding protein 5 (FKBP5) gene polymorphisms and their relationship with pharmacological response in bipolar disorders}

Braz J Psychiatry. 2021 Jan-Feb;43(1):111-112 doi:10.1590/1516-4446-2020-1231

\section{(cc) BY-NC}

Scaini et al. offer an informative review of current knowledge about the neurobiology of bipolar disorders, ${ }^{1}$ discussing the over-expression of FK506-binding protein 51 (FKBP51) as a putative mechanism conferring increased risk of mood dysregulation in bipolar disorders.

FKBP51 is involved in the hypothalamic pituitary axis feedback loop by acting as a co-chaperone for the sensitivity of the glucocorticoid receptor. ${ }^{1}$ This mechanism is of importance because FKBP51 could decrease the glucocorticoid receptor's affinity for circulating glucocorticoids and help explain the dysfunctional negative feedback of the hypothalamic pituitary axis, which could be responsible for the increased vulnerability to mood episodes in bipolar disorders. ${ }^{1,2}$

FKBP51 is expressed by the FKBP5 gene, which is located on chromosome $6 \mathrm{p} 21^{2,3}$ FKBP5 has been shown to be involved in the etiology of mood disorders, as well as in susceptibility to treatment response in mood disorders. ${ }^{2}$ Certain FKBP5 allele variants have been associated with exaggerated expression of the FKBP5 gene, resulting in FKBP51 over-expression. Three high induction alleles of major FKBP5 gene polymorphisms are believed to enhance FKBP5 mRNA expression following glucocorticoid receptor activation, including rs1360780 (T-allele), rs3800373 (C-allele), and rs4713916 (A-allele). ${ }^{3}$

In order to confirm previous reports ${ }^{3}$ of the role of FKBP5 high induction alleles, a systematic search of the literature on bipolar disorders, including PubMed, EMBASE, PsycINFO, Cochrane Library and Ovid MEDLINE, was conducted to identify studies that investigated FKBP5 polymorphisms in relation to treatment response in mood disorders. Studies were included if: 1) patients had mood disorders; 2) the disorders were diagnosed according to DSM or ICD classification systems; 3) the design was longitudinal; and 4) assessed response according to validated rating scales, expressed as odd ratios and $95 \%$ confidence intervals $(95 \% \mathrm{Cl})$. Eight studies met the criteria and included patients with unipolar and bipolar disorders, which allowed comparison between high and low induction alleles (detailed information available upon request via danilo.arnone@uaeu.ac. ae).

A random effects model meta-analysis was conducted in STATA to create a summary effect size and assess publication bias to investigate the relationship between high induction FKBP5 single nucleotide polymorphisms rs1360780, rs3800373, and rs4713916 and treatment response in mood disorders. The analysis of the rs4713916 FKBP5 A-high induction allele, obtained by combining three samples (two included patients with bipolar disorders), ${ }^{4,5}$ indicated that carriers of this polymorphism are more likely to respond to treatment in mood disorders (effect size: 1.28; $95 \% \mathrm{Cl} 1.04-1.57$ ). The analysis was free of heterogeneity $\left(\chi^{2}=1.01\right.$, degrees of freedom $=2, p=0.6$ ) and publication bias (Coef $=0.16, p=0.3$ ) (Figure 1). Analyses of the other two high induction alleles of FKBP5 polymorphisms showed no significant effect (rs1360780 effect size: 1.19; $95 \% \mathrm{Cl}$ 0.87-1.62; and rs3800373 effect size: 1.22 ; $95 \% \mathrm{Cl} 0.86-1.72)$ in the absence of publication bias (all p-values $>0.05$ ).

The results suggest that the high induction allele of rs4713916 in FKBP5 might increase susceptibility to treatment response in mood disorders. Further work is 